Vielerorts nimmt die Debatte über „Sozialwirtschaft“ ihren Ausgangspunkt bei konkreten Akteuren, insbesondere in der Gestalt von Nonprofit-Organisationen. Mit dieser apriorischen konzeptiven Verortung werden jedoch (implizit) Vorentscheidungen getroffen, die fragwürdig erscheinen. So wird nicht nur methodologisch-individualistisch die Summe von (korporativen) Akteuren als Startpunkt für empirische Deskription und Induktion genommen, sondern bleibt auch das lebensweltlich als oppositionell angesehene

\section{Was kann es heißen, von "Sozialwirtschaft" zu reden?}

Verhältnis zwischen dem Sozialen und der Wirtschaft weitgehend unbestimmt. Demgegenüber könnte wirklichkeitsnäher von Sozialwirtschaft als einem sozialsystemischen Zusammenhang, an dessen Hervorbringung und laufender Reproduktion individuelle wie korporative Akteure in ihrer jeweiligen Rolle als sozialwirtschaftliche Akteure beteiligt sind, ausgegangen werden. Damit ließe sich Sozialwirtschaft grundlegender fassen, was auch insofern nützlich wäre, als die Diskussion darüber verbreitet mit stark normativen Konzepten von Wirtschaft bzw. Wirtschaften (wirtschaftlichem Tun und Lassen) operiert, indem Orientierung am Leitwert der Effizienz als Kristallisationspunkt des Optimierungsstrebens (Maximierungs- bzw. Minimierungsprinzip) als Prämisse eingeführt wird. Allerdings ist wohl nicht länger einsichtig, weshalb ineffizientes Handeln (z.B. übermäßiger Ressourcenverbrauch oder das Zahlen überhöhter Preise) bzw. aufgrund der unterschiedlichen Systemreferenzen: aus Sicht des Akteurs anderweitig orientiertes Handeln nicht ebenfalls (unter anderem) wirtschaftliches Handeln darstellen sollte. Es geht also um Beobachtungen zumindest zweiter Ordnung.

Insofern ist es nützlich, erstens bei G. Simmel in dessen Philosophie des Geldes nachzulesen, wo Wirtschaften (und damit Wert-Schöpfung) über den in Tauschbeziehungen Handelnden bestimmt wird: Im Extremfall also sogar über ,jene[n] isolierte[n] Wirt, der gewisse Opfer zur Erzielung gewisser Früchte bringen muss ... nur dass sein Kontrahent nicht ein zweites wollendes Subjekt ist, sondern die natürliche Ordnung und Gesetzmäßigkeit der Dinge, die unsere Begehrungen - so wenig ohne ein Opfer unsrerseits zu erfüllen pflegt, wie ein anderer Mensch es tut“. Im offensichtlicheren und weitaus weniger strittigen Fall selbstverständlich durch die Realisierung des Tausches in der Form sozialer Wechselwirkungen, wenn dieses solipsistische ökonomische Handeln zu einem sozialen Handeln wird, aus dem sich jener systemische Zusammenhang bildet, dem sich (individuelle und kollektive) Akteure gegenübersehen: Wirtschaft als genuin soziales, d.h. gesellschaftliches Phänomen. Erst im Anschluss daran kann an die Frage herangegangen werden, was es bedeutet von „sozialem Wirtschaften“ bzw. von „Sozialwirtschaft“ zu reden.

Es kann ja wohl, zweitens, nicht darum gehen, von „sozialer Orientierung“ im umgangssprachlichen Sinne zu reden, also damit altruistische - im Unterschied zu egoistischer - Handlungsorientierung der Akteure zu bezeichnen. Falls überhaupt, so müsste von diesen beiden (am Nutzen Alters und Egos orientierten) Handlungstypen eine an sozialen bzw. moralischen Normen ausgerichtete unterschieden werden. Demnach würde eine „soziale Wirtschaft“, die alle möglichen (also auch: profitorientierte, erwerbswirtschaftliche) Aktivitäten umfassen kann, weil jedes Handeln innerhalb institutioneller Rahmen stets diese beiden Seiten der Medaille hat (wie von T. Parsons anhand der Motivierung wirtschaftlichen Handelns gezeigt), als emergentes Phänomen aus Tauschprozessen mit Blick auf den „moralischen Horizont“ (P. Strasser) entstehen.

Möglicherweise geht es allerdings, drittens, eher darum, dass die Rede von einer „Sozialwirtschaft“ soziale Wechselwirkungen unter dem Aspekt des Tauschens betrachten will. Dies jedoch nicht immer und überall (z.B. sensu P. M. Blau), sondern dort, wo durch angewandte Sozialwissenschaften bzw. „praktische Soziologie“ (H. Kern) jene Probleme, die das Individuum und die Gesellschaft als „ärgerliche Tatsache“ (R. Dahrendorf) sich wechselseitig bereiten, gelöst werden. Letztendlich also: Dass die Erzeugung Sozialer (nicht: pädagogischer, pflegerischer etc.) Arbeit für die „Gesellschaft der Individuen“ (N. Elias) - durch welche Akteure auch immer - mit Bezug auf deren Nutzen für KlientInnen, KundInnen und andere Anspruchsgruppen erklärt wird. Dies kommt letztendlich auch in der Bedeutung des Konzepts „soziale Dienstleistungen“ als Brenn- und Angelpunkt einer utilitaristischen Erklärung Sozialer Arbeit zum Ausdruck. 